\title{
MATRICES OVER POLYNOMIAL RINGS
}

\author{
BY \\ DAVID LISSNER( $\left.{ }^{1}\right)$
}

1. Introduction. The polynomial rings of the title are the rings of polynomials in a finite number of variables with coefficients in a field. In a paper [2] published in 1955, J.-P. Serre asked the question, is every finitely generated projective module over such a ring free? It is an easy exercise to show that this is the case for polynomials in one variable; in a recent article [3] C. S. Seshadri has shown that it is also true for polynomials in two variables. Otherwise the question remains open today, and it is perhaps à propos to remark that Stephen Chase of the University of Chicago has shown that the statement (that every finitely generated projective module is free) may be true for a ring $R$ and still fail to be true for $R[x]$.

The problem is equivalent to the following theorem: Let $R$ be a polynomial ring, $a_{1}, \cdots, a_{n} \in R$, and $\left(a_{1}, \cdots, a_{n}\right)=(1)$. Then $\exists$ an $n \times n$ matrix $M$, with entries in $R$, first row $\left(a_{1} \cdots a_{n}\right)$, and $|M|(=\operatorname{det} M)=1$.

(A proof of this equivalence will be given in §II. This proof has been known to Serre, and also to Kaplansky, for some time; it was communicated orally to the author by Professor Kaplansky. We include it here for completeness since it has not previously appeared in print.)

The purpose of this paper is to study a special case of this matrix theorem, and a few similar theorems closed related to it. Specifically, we will attempt to determine for which polynomial rings $R$ the following theorems hold.

TheOREM A. If $1 \in\left(a_{1}, a_{2}, a_{3}\right)$ then $\exists a 3 \times 3$ matrix $M$ over $R$ with first row $\left(a_{1} a_{2} a_{3}\right)$ and $|M|=1$.

Theorem B. If $d \in\left(a_{1}, a_{2}, a_{3}\right)$, where $d$ is any element of $R$, then $\exists a 3 \times 3$ matrix $M$ over $R$ with first row $\left(a_{1} a_{2} a_{3}\right)$ and $|M|=d$.

Theorem C. If $1 \in\left(a_{1}, a_{2}, a_{3}\right)$, then $\exists 2 \times 2$ matrices $A$ and $B$ over $R$ such that

$$
\left(\begin{array}{rr}
a_{1} & a_{2} \\
a_{3} & -a_{1}
\end{array}\right)=A B-B A .
$$

Received by the editors March 28, 1960.

(1) This work is, to within a few minor changes, the author's doctoral thesis, written at Cornell University for Professor I. N. Herstein. Part of the work was done while the author held a NSF pre-doctoral fellowship at Cornell; the paper was completed and submitted for publication during the tenure of an ONR post-doctoral fellowship at Northwestern University. I would like to take this opportunity to express my gratitude to Professor Herstein for his patience and friendly encouragement. 
Theorem D. If $C$ is any $2 \times 2$ matrix over $R$ with trace 0 , then $\exists 2 \times 2$ matrices $A$ and $B$ over $R$ such that $C=A B-B A$.

In the course of the paper we will show that for integral domains Theorems $A$ and $C$ are equivalent, and that $D$ implies $B$ for any commutative ring; thus $\mathrm{D}$ implies all the others. We also obtain the following results:

Theorem $\mathrm{D}$ is true for the ring of polynomials in one variable over any field, and for polynomials in two variables over any algebraically closed or real closed field. Thus in these cases all the theorems hold.

Theorem $\mathrm{D}$ is false for polynomials in three or more variables over any field.

Theorem B is false for polynomials in three or more variables over a formally real field, and for six or more variables over any field.

From the result of Seshadri already mentioned it follows that Theorem A (and hence $\mathrm{C}$ ) is true for two variables over any field. For three or more variables over any field these theorems remain open.

The analogue of Theorem $\mathrm{D}$ for $n \times n$ matrices is false for polynomials in $k$ variables over any field if $k$ is sufficiently large.

The method of approach will be to study Theorem D in detail, and derive results on the other theorems as corollaries whenever possible; e.g., it is the existence of a large class of counterexamples to Theorem $\mathrm{D}$ that enables us to produce a counterexample to Theorem $\mathrm{B}$.

2. Background. We give here the proof of the equivalence mentioned in the introduction.

Suppose first that every finitely generated projective module over the ring $R$ is free, and for this part of the proof we need only suppose that $R$ is any ring with unit for which the dimension of a free module is uniquely defined; in particular, $R$ may be any integral domain. Now given $a_{1}, \cdots, a_{n}$ $\in R$, with $\left(a_{1}, \cdots, a_{n}\right)=(1)$, we will construct the desired matrix. For this purpose, let $F$ be a free $R$-module of dimension $n$, with basis $\left\{u_{1}, \cdots, u_{n}\right\}$, and define a homomorphism $f: F \rightarrow R$ by $f\left(u_{i}\right)=a_{i}$ for all $i$. Then $f$ is onto, so we have an exact sequence

$$
0 \rightarrow K \rightarrow F \stackrel{f}{\rightarrow} R \rightarrow 0
$$

which splits, since $R$ is free. Thus $K$ is projective, and finitely generated, since it is a homomorphic image of $F$, and hence free. From the uniqueness of the dimension of $F$ it follows that $K$ has a basis $\left\{v_{1}, \cdots, v_{n-1}\right\}$ of $n-1$ generators, so that $\left\{v_{1}, \cdots, v_{n-1}, 1\right\}$ is a basis for $F=K \oplus R$. If we represent the $u_{i}^{\prime}$ 's in terms of this basis we have

$$
u_{i}=\sum_{j=1}^{n-1} a_{j i} v_{j}+a_{i}
$$


for each $i$. The matrix of coefficients is the required nonsingular matrix with last row $\left(a_{1} \cdots a_{n}\right)$.

Now suppose that $R$ is a polynomial ring for which the matrix theorem is true. It has been shown by Serre [6, exposé 23, p. 12] that every projective module over a polynomial ring has a free complement; i.e., if $P$ is projective then there are free modules $F_{1}$ and $F_{2}$ such that $F_{1}=F_{2} \oplus P$. It is a consequence of the proof that when $P$ is finitely generated $F_{1}$ and $F_{2}$ may also be taken to be finitely generated. Then by doing an induction on the dimension of $F_{2}$ we see that it will be sufficient to prove that if $R \oplus P$ is free then $P$ is free. Thus let $f: F \rightarrow R \oplus P$ be an isomorphism, where $F$ is a free module with basis $\left\{u_{1}, \cdots, u_{n}\right\}$, and let $f\left(u_{i}\right)=a_{i}+p_{i}$ for each $i$. Then since $f$ is onto $\left(a_{1}, \cdots, a_{n}\right)=(1)$, and so there exists a nonsingular $n \times n$ matrix $M$, with first row $\left(a_{1} \cdots a_{n}\right)$. M defines an isomorphism $m: F \rightarrow F$ by

$$
m\left(u_{i}\right)=a_{\imath} u_{1}+\sum_{j=2}^{n} a_{i j} u_{j}
$$

where the $a_{i j}$ 's are the remaining entries of $M$. Now define $\pi_{1}: F \rightarrow R$ by $\pi_{1}\left(\sum r_{i} u_{i}\right)=r_{1}$, and let $\pi_{R}$ be the projection of $R \oplus P$ onto $R$ along $P$. Then $\pi_{1} m: F \rightarrow R$ and $\pi_{R} f: F \rightarrow R$ agree on the $u_{i}$ 's and hence on all of $F$, and so have the same kernel, $K$. Now $m: K \rightarrow u_{2} R \oplus \cdots \oplus u_{n} R$ and $f: K \rightarrow P$ are both isomorphisms, so $m f^{-1}: P \rightarrow u_{2} R \oplus \cdots \oplus u_{n} R$ is also an isomorphism, as required.

3. Preliminaries. For this section, unless otherwise specified, $R$ will denote an arbitrary commutative ring with unit and $R_{2}$ the ring of $2 \times 2$ matrices over $R$. For $A, B \in R_{2}$ we will use $[A, B]$ to denote, as usual, the expression $A B-B A$. We observe that if $C=[A, B]$ and $[D, B]=0$, then $C=[A+D, B]$ as well, so we may add to $A$ anything that commutes with $B$, in particular any scalar matrix or any scalar multiple of $B$, without changing the commutator of $A$ and $B$. Then by adding a scalar matrix if necessary we can reduce $A$ to the form

$$
\left(\begin{array}{ll}
a_{1} & a_{2} \\
a_{3} & 0
\end{array}\right)
$$

and similarly for $B$. Thus a matrix $C$ is a commutator if and only if it can be expressed in the form

$$
C=\left(\begin{array}{cc}
c_{1} & c_{2} \\
c_{3} & -c_{1}
\end{array}\right)=\left[\left(\begin{array}{cc}
a_{1} & a_{2} \\
a_{3} & 0
\end{array}\right),\left(\begin{array}{cc}
b_{1} & b_{2} \\
b_{3} & 0
\end{array}\right)\right] ;
$$

i.e., if and only if $\exists a_{i}, b_{i} \in R$ satisfying the equations:

$$
\begin{aligned}
& c_{1}=a_{2} b_{3}-b_{2} a_{3}, \\
& c_{2}=a_{1} b_{2}-b_{1} a_{2}, \\
& c_{3}=a_{3} b_{1}-b_{3} a_{1} .
\end{aligned}
$$


Now let $\mathcal{K}$ be the set of all commutators in $R_{2}$. We record for future reference some manipulative lemmas.

LEMMA 3.1.

$$
\left(\begin{array}{cc}
c_{1} & c_{2} \\
c_{3} & -c_{1}
\end{array}\right) \in \mathfrak{K} \Rightarrow\left(\begin{array}{cc}
c_{i} & c_{j} \\
c_{k} & -c_{i}
\end{array}\right) \in \Re
$$

where $(i j k)$ is any permutation of (1 23 ).

Proof. It will be sufficient to verify that

$$
\left(\begin{array}{cc}
c_{1} & c_{3} \\
c_{2} & -c_{1}
\end{array}\right) \text { and }\left(\begin{array}{cc}
c_{2} & c_{3} \\
c_{1} & -c_{2}
\end{array}\right)
$$

are in $K$, since the permutations $\left(\begin{array}{lll}2 & 3\end{array}\right)$ and $\left(\begin{array}{lll}1 & 2 & 3\end{array}\right)$ generate $S_{3}$. Let

$$
\left(\begin{array}{cc}
c_{1} & c_{2} \\
c_{3} & -c_{1}
\end{array}\right)=[A, B]=\left[\left(\begin{array}{ll}
a_{1} & a_{2} \\
a_{3} & 0
\end{array}\right),\left(\begin{array}{ll}
b_{1} & b_{2} \\
b_{3} & 0
\end{array}\right)\right],
$$

and let $X^{r}$ denote the transpose of $X$. Then

$$
\left(\begin{array}{cc}
c_{1} & c_{3} \\
c_{2} & -c_{1}
\end{array}\right)=\left(\begin{array}{cc}
c_{1} & c_{2} \\
c_{3} & -c_{1}
\end{array}\right)^{T}=(A B-B A)^{T}=B^{T} A^{T}-A^{T} B^{T} \in \Re,
$$

and it follows from equations (1), (2), and (3) that

$$
\left(\begin{array}{cc}
c_{2} & c_{3} \\
c_{1} & -c_{2}
\end{array}\right)=\left[\left(\begin{array}{cc}
a_{3} & a_{1} \\
a_{2} & 0
\end{array}\right),\left(\begin{array}{ll}
b_{3} & b_{1} \\
b_{2} & 0
\end{array}\right)\right] .
$$

LEMMA 3.2.

$$
\left(\begin{array}{cc}
c_{1} & c_{2} \\
c_{3} & -c_{1}
\end{array}\right) \in \Re \Rightarrow\left(\begin{array}{rr}
e c_{1} & c_{2} \\
c_{3} & -e c_{1}
\end{array}\right) \in \Re,
$$

where $e$ is any unit in $R$.

Proof.

$$
\left(\begin{array}{rr}
c_{1} & c_{2} \\
c_{3} & -c_{1}
\end{array}\right) \in \Re
$$

implies that $\exists a_{i}, b_{i} \in R$ satisfying equations (1), (2), and (3), and it follows from these equations that

$$
\left(\begin{array}{cc}
e c_{1} & c_{2} \\
e_{3} & -e c_{1}
\end{array}\right)=\left[\left(\begin{array}{cc}
a_{1} & e a_{2} \\
e a_{3} & 0
\end{array}\right),\left(\begin{array}{cc}
e^{-1} b_{1} & b_{2} \\
b_{3} & 0
\end{array}\right)\right]
$$


LEMMA 3.3.

$$
\left(\begin{array}{rr}
c_{1} & c_{2} \\
c_{3} & -c_{1}
\end{array}\right) \in \mathcal{K} \Rightarrow\left(\begin{array}{rr}
c_{1} & d c_{2} \\
d c_{3} & -c_{1}
\end{array}\right) \in \mathcal{K}
$$

for any $d \in R$.

Proof. Again, it follows from equations (1), (2), and (3) that

$$
\left(\begin{array}{cc}
c_{1} & d c_{2} \\
d c_{3} & -c_{1}
\end{array}\right)=\left[\left(\begin{array}{cc}
d a_{1} & a_{2} \\
a_{3} & 0
\end{array}\right),\left(\begin{array}{cc}
d b_{1} & b_{2} \\
b_{3} & 0
\end{array}\right)\right] .
$$

LEMMA 3.4 .

$$
c_{1} \in\left(c_{2}, c_{3}\right) \Rightarrow\left(\begin{array}{cc}
c_{1} & c_{2} \\
c_{3} & -c_{1}
\end{array}\right) \in \Re
$$

Proof. Let $c_{1}=\alpha c_{2}+\beta c_{3}$. Then

$$
\left(\begin{array}{cc}
c_{1} & c_{2} \\
c_{3} & -c_{1}
\end{array}\right)=\left[\left(\begin{array}{rr}
0 & c_{2} \\
-c_{3} & 0
\end{array}\right),\left(\begin{array}{rr}
-1 & \beta \\
\alpha & 0
\end{array}\right)\right] .
$$

THEOREM 3.1. Let $R$ be any principal ideal ring, $C \in R_{2}$. Then trace $C=0 \Rightarrow C$ $\in \mathcal{K}$. (I.e., Theorem D holds for principal ideal rings.)

Proof. Let

$$
C=\left(\begin{array}{cc}
c_{1} & c_{2} \\
c_{3} & -c_{1}
\end{array}\right)
$$

and $\left(c_{2}, c_{3}\right)=(d)$, so that $c_{2}=d c_{2}^{\prime}, c_{3}=d c_{3}^{\prime}$, and $\left(c_{2}^{\prime}, c_{3}^{\prime}\right)=(1)$. Then $c_{1} \in\left(c_{2}^{\prime}, c_{3}^{\prime}\right)$, so

$$
\left(\begin{array}{rr}
c_{1} & c_{2}^{\prime} \\
c_{3}^{\prime} & -c_{1}
\end{array}\right) \in \mathscr{K}
$$

by Lemma 3.4 and so

$$
\left(\begin{array}{rr}
c_{1} & c_{2} \\
c_{3} & -c_{1}
\end{array}\right)=\left(\begin{array}{rr}
c_{1} & d c_{2}^{\prime} \\
d c_{3}^{\prime} & -c_{1}
\end{array}\right) \in \mathcal{K}
$$

by Lemma 3.3.

Equations (1), (2), and (3), are awkward to work with, but fortunately two of these can be replaced by the following two equations:

$$
\begin{aligned}
& 0=a_{1} c_{1}+a_{2} c_{3}+a_{3} c_{2} \\
& 0=b_{1} c_{1}+b_{2} c_{3}+b_{3} c_{2} .
\end{aligned}
$$

The precise situation is given by the next two lemmas. 
Lemma 3.5. If $R$ is an integral domain and $c_{1} \neq 0$, then equations (1), (2) and (3) are equivalent to equations (1), (4), and (5), in the sense that the $a_{i}^{\prime}$ 's and $b_{i}$ 's satisfy one set of equations if and only if they satisfy the other.

Proof. Suppose we have $a_{i}, b_{i}$ satisfying equations (1), (2), and (3). Then

$$
\begin{aligned}
a_{1} c_{1}+a_{2} c_{3}+a_{3} c_{2} & =a_{1}\left(a_{2} b_{3}-b_{2} a_{3}\right)+a_{2}\left(a_{3} b_{1}-b_{3} a_{1}\right)+a_{3}\left(a_{1} b_{2}-b_{1} a_{2}\right) \\
& =0,
\end{aligned}
$$

and similarly for equation (5).

Now suppose the $a_{i}, b_{i}$ satisfy equations (1), (4), and (5). Multiplying equation (4) by $b_{3}$ and (5) by $a_{3}$ and subtracting, we get

$$
0=\left(a_{1} b_{3}-b_{1} a_{3}\right) c_{1}+\left(a_{2} b_{3}-b_{2} a_{3}\right) c_{3},
$$

which is equivalent to

$$
\left(a_{3} b_{1}-b_{3} a_{1}\right) c_{1}=c_{1} c_{3}
$$

by equation (1). Now since $c_{1} \neq 0$ and $R$ is an integral domain, this gives us equation (3), and (2) may be obtained in the same way.

Lemma 3.6. If $R$ is an integral domain, then

$$
\left(\begin{array}{cc}
c_{1} & c_{2} \\
c_{3} & -c_{1}
\end{array}\right) \in \Re \Leftrightarrow \exists a_{i}, b_{i} \in \Re
$$

satisfying equations (1), (4), and (5).

Proof. If $c_{1}=0$ we certainly have $c_{1} \in\left(c_{2}, c_{3}\right)$, so that

$$
\left(\begin{array}{cc}
c_{1} & c_{2} \\
c_{3} & -c_{1}
\end{array}\right) \in \Re
$$

by Lemma 3.4, and otherwise Lemma 3.5 is applicable. This gives the implication in one direction. For the other, we note that in the previous lemma the proof of the fact that equations (1), (2), and (3) imply equations (1), (4), and (5) did not depend on $c_{1} \neq 0$.

Lemma 3.7. If $R$ is an integral domain, and $\alpha c_{1}+\beta c_{2}+\gamma c_{3}=1$, then

$$
\left(\begin{array}{cc}
c_{1} & c_{2} \\
c_{3} & -c_{1}
\end{array}\right) \in \mathcal{K} \Leftrightarrow\left(\begin{array}{cc}
\alpha & \beta \\
\gamma & -\alpha
\end{array}\right) \in \Re .
$$

Proof. It is certainly sufficient to prove the implication in one direction only, so suppose that

$$
\left(\begin{array}{rr}
\alpha & \beta \\
\gamma & -\alpha
\end{array}\right) \in \Re .
$$

We will then produce $a_{i}, b_{i} \in R$ satisfying equations (1), (4), and (5). Let $R^{8}$ 
denote the free $R$-module on three generators, $N$ the submodule consisting of all triples $\left(r_{1}, r_{2}, r_{3}\right)$ satisfying

$$
0=r_{1} c_{1}+r_{2} c_{3}+r_{3} c_{2}
$$

and $N^{\prime}$ the submodule generated by the three vectors

$$
\begin{aligned}
& \bar{u}_{1}=\left(0,-c_{2}, c_{3}\right), \\
& \bar{u}_{2}=\left(-c_{2}, 0, c_{1}\right),
\end{aligned}
$$

and

$$
\bar{u}_{3}=\left(-c_{3}, c_{1}, 0\right) .
$$

Certainly $N^{\prime} \subseteq N$. The idea is to exhibit vectors $\bar{a}=\left(a_{1}, a_{2}, a_{3}\right)$ and $\bar{b}=\left(b_{1}, b_{2}, b_{3}\right)$ in $N^{\prime}$ whose coordinates satisfy equation (1); equations (4) and (5) will then be satisfied automatically. Let

$$
\bar{a}=k_{1} \bar{u}_{1}+k_{2} \bar{u}_{2}+k_{3} \bar{u}_{3} \text { and } \quad \bar{b}=m_{1} \bar{u}_{1}+m_{2} \bar{u}_{2}+m_{3} \bar{u}_{3} .
$$

Then

$$
\begin{array}{ll}
a_{2}=-k_{1} c_{2}+k_{3} c_{1}, & a_{3}=k_{1} c_{3}+k_{2} c_{1}, \\
b_{2}=-m_{1} c_{2}+m_{3} c_{1}, & \text { and } \quad b_{3}=m_{1} c_{3}+m_{2} c_{1},
\end{array}
$$

so that the equation we must satisfy becomes

$$
\begin{aligned}
c_{1} & =a_{2} b_{3}-b_{2} a_{3} \\
& =\left(-k_{1} c_{2}+k_{3} c_{1}\right)\left(m_{1} c_{3}+m_{2} c_{1}\right)-\left(-m_{1} c_{2}+m_{3} c_{1}\right)\left(k_{1} c_{3}+k_{2} c_{1}\right) \\
& =\left[\left(m_{1} k_{2}-k_{1} m_{2}\right) c_{2}+\left(k_{3} m_{1}-m_{3} k_{1}\right) c_{3}+\left(k_{3} m_{2}-m_{3} k_{2}\right) c_{1}\right] c_{1} .
\end{aligned}
$$

This will certainly be satisfied if $k_{3} m_{2}-m_{3} k_{2}=\alpha, m_{1} k_{2}-k_{1} m_{2}=\beta$, and $k_{3} m_{1}$ $m_{3} k_{1}=\gamma$. We claim that since

$$
\left(\begin{array}{rr}
\alpha & \beta \\
\gamma & -\alpha
\end{array}\right)
$$

is a commutator we can accomplish this. Indeed, -1 is always a unit, so by Lemmas 3.1 and 3.2

$$
\left(\begin{array}{rr}
\alpha & \beta \\
-\gamma & -\alpha
\end{array}\right)
$$

is also a commutator, and we need only choose the $m_{i}^{\prime}$ 's and $k_{i}$ 's so that

$$
\left(\begin{array}{rr}
\alpha & \beta \\
-\gamma & -\alpha
\end{array}\right)=\left[\left(\begin{array}{cc}
m_{1} & m_{2} \\
m_{3} & 0
\end{array}\right),\left(\begin{array}{ll}
k_{1} & k_{2} \\
k_{3} & 0
\end{array}\right)\right]
$$

THEOREM 3.2. If $R$ is an integral domain, Theorems $\mathrm{A}$ and $\mathrm{C}$ are equivalent. 
Proof. Suppose first that Theorem $A$ is true, and $1 \in\left(a_{1}, a_{2}, a_{3}\right)$. Then there exist $k_{i}, m_{i}$ such that

$$
\left|\begin{array}{ccc}
a_{1} & a_{3} & a_{2} \\
k_{1} & k_{2} & k_{3} \\
m_{1} & m_{2} & m_{3}
\end{array}\right|=\left(k_{2} m_{3}-m_{2} k_{3}\right) a_{1}+\left(k_{1} m_{2}-m_{1} k_{2}\right) a_{2}+\left(k_{3} m_{1}-m_{3} k_{1}\right) a_{3}
$$

now

$$
\left(\begin{array}{rr}
\alpha & \beta \\
\gamma & -\alpha
\end{array}\right)
$$

is certainly a commutator, and so, by the previous lemma,

$$
\left(\begin{array}{rr}
a_{1} & a_{2} \\
a_{3} & -a_{1}
\end{array}\right)
$$

is a commutator also.

Now suppose that Theorem $\mathrm{C}$ is true, and that $1 \in\left(a_{1}, a_{2}, a_{3}\right)$. Then there are $\alpha, \beta, \gamma \in R$ with $\alpha a_{1}+\beta a_{2}+\gamma a_{3}=1$; thus $1 \in(\alpha, \beta, \gamma)$ as well, so applying Theorem $\mathrm{C}$ to this case we have that there exist $k_{i}, m_{i} \in R$ such that

$$
\left(\begin{array}{cc}
\alpha & \beta \\
\gamma & -\alpha
\end{array}\right)=\left[\left(\begin{array}{cc}
k_{1} & k_{2} \\
k_{3} & 0
\end{array}\right),\left(\begin{array}{cc}
m_{1} & m_{2} \\
m_{3} & 0
\end{array}\right)\right] \text {. }
$$

Then

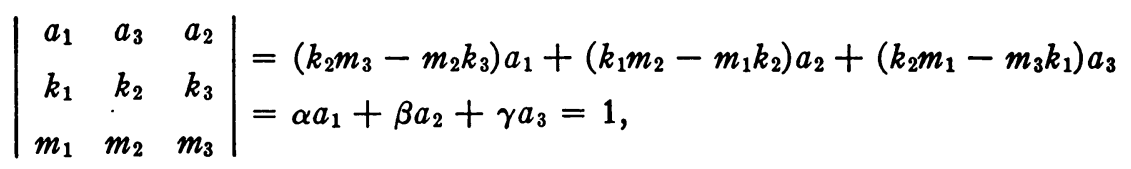

as required.

If in the last part of this proof we replace the condition " $1 \in\left(a_{1}, a_{2}, a_{3}\right)$ " with " $d \in\left(a_{1}, a_{2}, a_{3}\right)$ " and assume that every $2 \times 2$ matrix of trace 0 over $R$ is a commutator, it is clear we can construct a $3 \times 3$ matrix with first row $\left(a_{1} a_{3} a_{2}\right)$ and determinant $d$. Furthermore, in this part of the proof we haven't used the fact that $R$ is an integral domain, so we have shown

TheOREM 3.3. For any commutative ring, Theorem D implies Theorem B.

Lemma 3.8. Let $R$ be an integral domain, and $p \in\left(c_{1}, c_{2}\right)$. Then

$$
\left(\begin{array}{cc}
c_{1} & c_{2} \\
c_{3} & -c_{1}
\end{array}\right) \in \mathscr{K} \Leftrightarrow\left(\begin{array}{cc}
c_{1} & c_{2} \\
c_{3}+p & -c_{1}
\end{array}\right) \in \mathcal{K} .
$$

Proof. It is certainly sufficient to prove the implication in one direction, and for this purpose it will suffice to show that for any $r \in R$, 


$$
\left(\begin{array}{cc}
c_{1} & c_{2} \\
c_{3} & -c_{1}
\end{array}\right) \in \Re \Rightarrow\left(\begin{array}{cc}
c_{1} & c_{2} \\
c_{3}+r c_{1} & -c_{1}
\end{array}\right) \in \mathfrak{K} ;
$$

since $p$ can be written as $r c_{1}+s c_{2}$ the lemma will then follow by applying this fact twice, and using Lemma 3.1.

Now the hypothesis implies that $\exists a_{i}, b_{i} \in R$ such that

$$
\begin{aligned}
c_{1} & =a_{2} b_{3}-b_{2} a_{3} \\
0 & =a_{1} c_{1}+a_{2} c_{3}+a_{3} c_{2}
\end{aligned}
$$

and

$$
0=b_{1} c_{1}+b_{2} c_{3}+b_{3} c_{2}
$$

From the last two equations we get

$$
0=\left(a_{1}-r a_{2}\right) c_{1}+a_{2}\left(c_{3}+r c_{1}\right)+a_{3} c_{2}
$$

and

$$
0=\left(b_{1}-r b_{2}\right) c_{1}+b_{2}\left(c_{3}+r c_{1}\right)+b_{3} c_{2},
$$

and these, plus the first equation above, give

$$
\left(\begin{array}{cr}
c_{1} & c_{2} \\
c_{3}+r c_{1} & -c_{1}
\end{array}\right) \in \Re
$$

by Lemma 3.6.

From here on we will assume that $R$ is a unique factorization domain, that is, an integral domain in which every element may be factored uniquely (to within units) into a product of prime elements. Given $a, b \in R$, we will use $\langle a, b\rangle$ to denote the greatest common factor of $a$ and $b$ (again, unique to within multiplication by a unit); in particular, we write $\langle a, b\rangle=1$ if $a$ and $b$ have no common factors other than units.

The next lemma gives us a partial converse to Lemma 3.3 which allows us to restrict our attention to a smaller class of matrices.

LEMMA 3.9. If $\left\langle k, c_{1}\right\rangle=1$, then

$$
\left(\begin{array}{cc}
d c_{1} & k d c_{2} \\
k d c_{3} & -d c_{1}
\end{array}\right) \in \Re \Rightarrow\left(\begin{array}{cc}
d c_{1} & d c_{2} \\
d c_{3} & -d c_{1}
\end{array}\right) \in \mathfrak{K} .
$$

Proof. The hypothesis implies that $\exists a_{i}, b_{i} \in R$ satisfying

$$
\begin{aligned}
d c_{1} & =a_{2} b_{3}-b_{2} a_{3} \\
0 & =a_{1}\left(d c_{1}\right)+a_{2}\left(k d c_{3}\right)+a_{3}\left(k d c_{2}\right)
\end{aligned}
$$

and 


$$
0=b_{1}\left(d c_{1}\right)+b_{2}\left(k d c_{3}\right)+b_{3}\left(k d c_{2}\right)
$$

We may assume $d \neq 0$ (since otherwise there is nothing to prove), so the last two equations give

$$
0=a_{1} c_{1}+a_{2} k c_{3}+a_{3} k c_{2}
$$

and

$$
0=b_{1} c_{1}+b_{2} k c_{3}+b_{3} k c_{2} .
$$

Thus $k \mid a_{1} c_{1}$ and $k \mid b_{1} c_{1}$. But since $\left\langle k, c_{1}\right\rangle=1$, this means that $k \mid a_{1}$ and $k \mid b_{1}$; let $a_{1}=k a_{1}^{\prime}$ and $b_{1}=k b_{1}^{\prime}$. Now factoring $k$ out of the last two equations $\left(k \neq 0\right.$ is implicit in $\left.\left\langle k, c_{1}\right\rangle=1\right)$ and multiplying by $d$ gives

$$
0=a_{1}^{\prime}\left(d c_{1}\right)+a_{2}\left(d c_{3}\right)+a_{3}\left(d c_{2}\right)
$$

and

$$
0=b_{1}^{\prime}\left(d c_{1}\right)+b_{2}\left(d c_{3}\right)+b_{3}\left(d c_{2}\right) .
$$

These two equations, plus the first equation above, imply that

$$
\left(\begin{array}{rr}
d c_{1} & d c_{2} \\
d c_{3} & -d c_{1}
\end{array}\right) \in \mathcal{K}
$$

by Lemma 3.6.

Now let

$$
A=\left(\begin{array}{rr}
a_{1} & a_{2} \\
a_{3} & -a_{1}
\end{array}\right)
$$

be any $2 \times 2$ matrix of trace 0 . If any of the $a_{i}$ 's is 0 we know that $A$ is a commutator, so we may suppose that each $a_{i} \neq 0$. Then if $d=\left\langle a_{1}, a_{2}, a_{3}\right\rangle$ (the greatest factor common to all three $a_{i}$ 's) we have

$$
A=\left(\begin{array}{cc}
d b_{1} & d b_{2} \\
d b_{3} & -d b_{1}
\end{array}\right) \text { where }\left\langle b_{1}, b_{2}, b_{3}\right\rangle=1 .
$$

Using the previous lemma together with Lemma 3.3 we can now remove any factors common to two of the $b_{i}$ 's; e.g., if $b_{2}=k c_{2}$ and $b_{3}=k c_{3}$ then $\left\langle k, b_{1}\right\rangle=1$ so $A$ is a commutator if and only if

$$
\left(\begin{array}{cc}
d b_{1} & d c_{2} \\
d c_{3} & -d b_{1}
\end{array}\right)
$$

is. Proceeding in this way we construct a matrix

$$
A^{*}=\left(\begin{array}{cc}
d c_{1} & d c_{2} \\
d c_{3} & -d c_{1}
\end{array}\right)
$$

with the property $\left\langle c_{i}, c_{j}\right\rangle=1$ for all $i$ and $j$, and $A^{*} \in \mathscr{K}$ if and only if $A \in \mathscr{K}$. 
Thus the general problem reduces to the consideration of matrices of the form $d C$, where

$$
C=\left(\begin{array}{rr}
c_{1} & c_{2} \\
c_{3} & -c_{1}
\end{array}\right) \quad \text { and }\left\langle c_{i}, c_{j}\right\rangle=1 \text { for all } i \text { and } j .
$$

This divides itself naturally into two questions. First, what matrices of this type (i.e., no two of whose entries have common factors) are commutators, and second, given a matrix $C \notin \mathcal{K}$, for what $d$ will $d C \in \mathcal{K}$ ?

With regard to the second problem, we know that such $d$ 's always exist; e.g., by Theorem 3.1 $C$ is certainly a commutator if we allow matrices over the quotient field of $R$, and then if $d$ is the product of all the denominators occurring in such an expression, $d C$ will clearly be a commutator of matrices over $R$. The problem of determining precisely what $d$ 's will do the trick for a given matrix $C$ appears to be quite difficult, and of relatively little interest (remember that the problem which originally motivated this paper is the one for which the entries in the matrix generate the unit ideal, and hence have no common factors); we will accordingly restrict our attention to the first problem.

4. Polynomials in two variables. For this section, $R$ will be $K[x, y]$, $K$ an arbitrary field.

Lemma. If $\left\langle c_{1}, c_{2}\right\rangle=1$ and $p$ is a product of linear factors, then

$$
\left(\begin{array}{rr}
c_{1} & c_{2} \\
p c_{3} & -c_{1}
\end{array}\right) \in \mathscr{K} \Rightarrow\left(\begin{array}{cc}
c_{1} & c_{2} \\
c_{3} & -c_{1}
\end{array}\right) \in \mathscr{K} .
$$

Proof. It will be enough to prove this in the case where $p$ is linear; the lemma then follows by repeated application of this special case. For this purpose, it will be sufficient to prove the lemma for $p=x$, for we can reduce an arbitrary linear polynomial to $x$ by a linear change of variable. That is, if $p=a_{0}+a_{1} x+a_{2} y$ and $a_{1} \neq 0$, set $x^{\prime}=p, y^{\prime}=y$. Then $K[x, y]=K\left[x^{\prime}, y^{\prime}\right]$ and we can apply the lemma for $p=x^{\prime}$ to $K\left[x^{\prime}, y^{\prime}\right]$. If $a_{1}=0$ then $a_{2} \neq 0$ and we can take $x^{\prime}=p$ and $y^{\prime}=x$.

Thus we are reduced to the case $p=x$. Now since $\left\langle c_{1}, c_{2}\right\rangle=1$ either $x \nmid c_{1}$ or $x \nmid c_{2}$. By Lemma 3.1 we are justified in assuming that $x \nmid c_{2}$. Let

$$
\left(\begin{array}{rr}
c_{1} & c_{2} \\
x c_{3} & -c_{1}
\end{array}\right)=[A, B]=\left[\left(\begin{array}{ll}
a_{1} & a_{2} \\
a_{3} & 0
\end{array}\right),\left(\begin{array}{ll}
b_{1} & b_{2} \\
b_{3} & 0
\end{array}\right)\right],
$$

where $a_{1}=x p_{1}(x, y)+p_{0}(y)$ and $b_{1}=x q_{1}(x, y)+q_{0}(y)$, the $p_{i}$ 's and $q_{i}$ 's being polynomials in the indicated variables. Now we can write $p_{0}(y)$ as

$$
p_{0}(y)=r_{1}(y) q_{0}(y)+r_{2}(y),
$$

where either $r_{2}(y)=0$ or $\operatorname{deg} r_{2}<\operatorname{deg} q_{0}$. We know that we can add to $A$ any 
scalar multiple of $B$ without changing the commutator of $A$ and $B$; in particular

$$
\begin{aligned}
\left(\begin{array}{rr}
c_{1} & c_{2} \\
x c_{3} & -c_{1}
\end{array}\right) & =[A, B]=\left[A-r_{1} B, B\right] \\
& =\left[\left(\begin{array}{cc}
x p_{1}^{\prime}(x, y)+r_{2}(y) & a_{2}^{\prime} \\
a_{3}^{\prime} & 0
\end{array}\right),\left(\begin{array}{cc}
x q_{1}(x, y)+q_{0}(y) & b_{2} \\
b_{3} & 0
\end{array}\right)\right] .
\end{aligned}
$$

Now we can write $q_{0}$ as $r_{3} r_{2}+r_{4}$ and repeat the procedure, this time replacing $B$ by $B-r_{3}\left(A-r_{1} B\right)$, in the course of which $b_{1}$ is replaced by $x q_{1}^{\prime}(x, y)+r_{4}(y)$. It is clear that if we continue in this way we will eventually arrive at an $r_{k}=0$. We may suppose that this occurs in the matrix $A$, so that $a_{1}^{(j)}=x p_{1}^{(j)}(x, y)$ +0 , i.e., $a_{1}^{(j)}$ is a multiple of $x$. Thus we are actually justified in assuming that

$$
\left(\begin{array}{cc}
c_{1} & c_{2} \\
x c_{3} & -c_{1}
\end{array}\right)=\left[\left(\begin{array}{cc}
x a_{1} & a_{2} \\
a_{3} & 0
\end{array}\right),\left(\begin{array}{ll}
b_{1} & b_{2} \\
b_{3} & 0
\end{array}\right)\right] .
$$

Now equations (3), (4), and (5) of $\$ 3$ become

$$
\begin{aligned}
x c_{3} & =a_{3} b_{1}-b_{3}\left(x a_{1}\right), \\
0 & =\left(a_{1} x\right) c_{1}+a_{2}\left(x c_{3}\right)+a_{3} c_{2},
\end{aligned}
$$

and

$$
0=b_{1} c_{1}+b_{2}\left(x c_{3}\right)+b_{3} c_{2} .
$$

Since $x \nmid c_{2}$ the second equation implies that $x \mid a_{3} ;$ say $a_{3}=x a_{3}^{\prime}$. Then factoring an $x$ out of the first two equations gives

$$
c_{3}=a_{3}^{\prime} b_{1}-b_{3} a_{1}
$$

and

$$
0=a_{1} c_{1}+a_{2} c_{3}+a_{3}^{\prime} c_{2},
$$

while the last equation may be written as

$$
0=b_{1} c_{1}+\left(b_{2} x\right) c_{3}+b_{3} c_{2} .
$$

These last three equations imply that

$$
\left(\begin{array}{cc}
c_{1} & c_{2} \\
c_{3} & -c_{1}
\end{array}\right) \in \Re
$$

by Lemma 3.6.

ThEOREM 4.1. Let $R=K[x, y], K$ algebraically closed, and $C \in R_{2}$. Then $\operatorname{tr}(C)=0 \Rightarrow C \in \varkappa$.

Proof. We showed at the end of $\S 3$ that it is sufficient to prove the theorem for all matrices of the form $d C$, where 


$$
C=\left(\begin{array}{rr}
c_{1} & c_{2} \\
c_{3} & -c_{1}
\end{array}\right) \text { and }\left\langle c_{i}, c_{j}\right\rangle=1 \text { for all } i \text { and } j .
$$

For this purpose it will be sufficient to show that $C \in \mathcal{K}$, for then certainly $d C \in \mathcal{K}$ also. Now it is well known that since $\left\langle c_{1}, c_{2}\right\rangle=1$ there is a polynomial $p(x) \in\left(c_{1}, c_{2}\right)$ which is a function of $x$ only; e.g., we could take $p(x)$ to be the resultant of $c_{1}$ and $c_{2}$ considered as polynomials in $y$ with coefficients in $K[x]$; this is in $\left(c_{1}, c_{2}\right)$ (see van der Waerden $\left[4\right.$, p. 85]) and is not 0 since $\left\langle c_{1}, c_{2}\right\rangle=1$. Then $p(x) c_{3} \in\left(c_{1}, c_{2}\right)$ also, so

$$
\left(\begin{array}{cr}
c_{1} & c_{2} \\
p(x) c_{3} & -c_{1}
\end{array}\right) \in \mathscr{K}
$$

by Lemma 3.4. But since $K$ is algebraically closed, $p(x)$ splits into linear factors, and so

$$
\left(\begin{array}{cc}
c_{1} & c_{2} \\
c_{3} & -c_{1}
\end{array}\right) \in \mathscr{K}
$$

by the lemma just proved.

TheOREM 4.2. Let $R=K[x, y], K$ a real closed field, and $C \in R_{2}$. Then $\operatorname{tr}(C)=0 \Rightarrow C \in \Re$.

Proof. As above we may assume

$$
C=\left(\begin{array}{rr}
c_{1} & c_{2} \\
c_{3} & -c_{1}
\end{array}\right) \text { with }\left\langle c_{i}, c_{j}\right\rangle=1 \text { for all } i \text { and } j,
$$

and it will be sufficient to show that there is a polynomial $p \in\left(c_{1}, c_{2}\right)$ which is a product of linear factors. Let

$$
\left(c_{1}, c_{2}\right)=q_{1} \cap \cdots \cap q_{k},
$$

where each $\mathfrak{q}_{i}$ is a primary ideal with corresponding prime ideal $\mathfrak{p}_{i}$ and exponent $\alpha_{i}$ (that is, $\alpha_{i}$ is the least integer such that $\mathfrak{p}_{i}^{\alpha_{i}} \subseteq \mathfrak{q}_{i}$ ). It will be sufficient to show that there is a linear polynomial $p_{i}$ in each $\mathfrak{p}_{i}$, for then $p_{i}^{\alpha_{i}} \in \mathfrak{q}_{i}$ and

$$
p=\prod_{i} p_{i}^{\alpha_{i}} \in q_{1} \cap \cdots \cap q_{k}=\left(c_{1}, c_{2}\right)
$$

Now since $\left\langle c_{1}, c_{2}\right\rangle=1$ there is, as we have just observed, a polynomial $p(x)$ $\in\left(c_{1}, c_{2}\right)$, and similarly also a $q(y) \in\left(c_{1}, c_{2}\right)$. Let $\left(x_{0}, y_{0}\right)$ be a zero of the ideal $\left(c_{1}, c_{2}\right)$, i.e., a common zero of $c_{1}$ and $c_{2}$. Then $p\left(x_{0}\right)=0$ and $q\left(y_{0}\right)=0$, so there are only a finite number of possibilities for $x_{0}$ and $y_{0}$, and we have now shown that the manifold of $\left(c_{1}, c_{2}\right)$ consists of at most a finite number of points. If the manifold is empty $\left(c_{1}, c_{2}\right)=(1)$ and there is nothing to prove. Otherwise $\left(c_{1}, c_{2}\right)$ is a zero-dimensional ideal, and so the $\mathfrak{p}_{i}$ 's are zero-dimensional prime 
ideals. Thus the manifold of a $\mathfrak{p}_{i}$ consists of algebraically conjugate points. It will now be sufficient to produce a linear polynomial which is 0 at one of these points; since the points are conjugate it will then be 0 at all of them and hence be in $p_{i}$. Let $i$ be a root of the polynomial $x^{2}+1$, and let $\bar{K}=K[i]$. Then we know that $\bar{K}$ is the algebraic closure of $K$, and so a point in the manifold of $p_{i}$ has the form $\left(a_{1}+i a_{2}, b_{1}+i b_{2}\right)$, where the $a_{i}$ 's and $b_{i}$ 's are in $K$, and we have to find a linear polynomial $p_{i} \in K[x, y]$ which is 0 at this point. This is easy enough: if $b_{2} \neq 0$ take

$$
p_{i}=x-\left(\frac{a_{2}}{b_{2}}\right) y-\left(a_{1}-\frac{a_{2}}{b_{2}} b_{1}\right),
$$

otherwise take $p_{i}=y-b_{1}$.

5. Polynomials in more than two variables. From here on $R$ will be $K\left[x_{1}, \cdots, x_{n}\right]$, where $K$ is a field and $n \geqq 3$. In keeping with the remarks made at the end of $\S 3$, we will restrict ourselves to the consideration of matrices of the form

$$
C=\left(\begin{array}{cc}
c_{1} & c_{2} \\
c_{3} & -c_{1}
\end{array}\right), \text { where }\left\langle c_{i}, c_{j}\right\rangle=1 \text { for all } i \text { and } j .
$$

We recall a notation established earlier: $R^{3}$ is the free $R$-module on three generators, $N$ the submodule consisting of all triples $\left(r_{1}, r_{2}, r_{3}\right)$ such that

$$
0=r_{1} c_{1}+r_{2} c_{3}+r_{3} c_{2},
$$

and $N^{\prime}$ the submodule of $N$ generated by

$$
\begin{aligned}
& \bar{u}_{1}=\left(0,-c_{2}, c_{3}\right), \\
& \bar{u}_{2}=\left(-c_{2}, 0, c_{1}\right),
\end{aligned}
$$

and

$$
\bar{u}_{3}=\left(-c_{3}, c_{1}, 0\right) .
$$

Let $\bar{a}=\left(a_{1}, a_{2}, a_{3}\right)$ and $\bar{b}=\left(b_{1}, b_{2}, b_{3}\right)$. Then $C \in \Re$ if and only if $\exists \bar{a}, \bar{b} \in N$ whose coordinates satisfy $c_{1}=a_{2} b_{3}-b_{2} a_{3}$. It will thus be of interest to describe $N$ more precisely.

Let $\left(c_{1}, c_{2}\right)=q_{1} \cap \cdots \cap q_{k}$, where the $q_{i}$ 's are primary ideals with corresponding prime ideals $\mathfrak{p}_{i}$, and $\mathfrak{p}_{i} \neq \mathfrak{p}_{j}$ for $i \neq j$. Then we have

Lemma 5.1. If $c_{3} \notin p_{1} \cup \cdots \cup \mathfrak{p}_{k}$ then $N=N^{\prime}$.

Proof. We have $N^{\prime} \subseteq N$ in any case, so we must show that $N \subseteq N^{\prime}$. Let the vector $\bar{a}=\left(a_{1}, a_{2}, a_{3}\right) \in N$, so that

$$
0=a_{1} c_{1}+a_{2} c_{3}+a_{3} c_{2} .
$$

Then $a_{2} \in\left(c_{1}, c_{2}\right):\left(c_{3}\right)$; but the hypothesis implies that $\left(c_{1}, c_{2}\right):\left(c_{3}\right)=\left(c_{1}, c_{2}\right)$ (see Northcott [1]), so we actually have $a_{2} \in\left(c_{1}, c_{2}\right)$; say $a_{2}=k_{1} c_{1}+k_{2} c_{2}$. Then the above equation becomes 


$$
0=\left(a_{1}+k_{1} c_{3}\right) c_{1}+\left(a_{3}+k_{2} c_{3}\right) c_{2} .
$$

Now since $\left\langle c_{1}, c_{2}\right\rangle=1$ it follows that $c_{2} \mid\left(a_{1}+k_{1} c_{3}\right)$, say $a_{1}+k_{1} c_{3}=k_{3} c_{2}$, and similarly $a_{3}+k_{2} c_{3}=k_{4} c_{1}$. We then have

$$
a_{1}=k_{3} c_{2}-k_{1} c_{3}
$$

and

$$
a_{3}=k_{4} c_{1}-k_{2} c_{3}
$$

so our equation becomes

$$
0=\left(k_{3}+k_{4}\right) c_{1} c_{2} \text {. }
$$

Thus $k_{4}=-k_{3}$ (since we have restricted ourselves to a case in which none of the $c_{i}$ 's are 0$)$ so that

$$
a_{3}=-k_{3} c_{1}-k_{2} c_{3},
$$

and so $\left(a_{1}, a_{2}, a_{3}\right)=-k_{2} \bar{u}_{1}-k_{3} \bar{u}_{2}+k_{1} \bar{u}_{3} \in N^{\prime}$, as required.

We define the dimension of an ideal $I$, as usual, to be the dimension of its manifold, i.e., the maximum of the dimensions of its prime ideals, and recall the following properties of this dimension:

(1) $\operatorname{dim} I=-1$ is defined to mean that the manifold of $I$ is empty, i.e., that $I=R$,

(2) $\operatorname{dim} I=n-1$ if and only if $I$ is a principal ideal (see van der Waerden $[5$, p. 68 , exercise 2$])$,

(3) If $I$ is generated by $r$ elements, and $I \neq R$, then $\operatorname{dim} I \geqq n-r$ (see Northcott $[1$, p. 60 , Theorem 7$])$.

Now let $I(C)$ denote the ideal $\left(c_{1}, c_{2}, c_{3}\right)$. Since the $c_{i}$ 's have no common factors $I(C)$ is not a principal ideal (unless $I(C)=R$ ), hence $\operatorname{dim} I(C)$ is either $-1, n-3$, or $n-2$. The case where $\operatorname{dim} I(C)$ is -1 , i.e., where $I(C)=R$, is just Theorem $\mathrm{C}$, which is still unsettled. When $\operatorname{dim} I(C)=n-3$ we have the following:

Theorem 5.1. Let $R=K\left[x_{1}, \cdots, x_{n}\right], n \geqq 3, K$ any field, and let $C \in R_{2}$, with $\operatorname{tr}(C)=0$. If $\operatorname{dim} I(C)=n-3$ then $C \notin \Re$.

Proof. We first note that since $n \geqq 3 \operatorname{dim} I(C) \neq-1$, i.e., $I(C) \neq R$.

Now we claim that the hypothesis of Lemma 5.1 is satisfied, that is, $c_{3} \notin p_{1} \cup \ldots \cup \mathfrak{p}_{k}$, where the $\mathfrak{p}_{i}$ 's are the prime ideals belonging to $\left(c_{1}, c_{2}\right)$. For if $c_{3} \in p_{i}$ for some $i$, then $I(C) \subseteq p_{i}$, and so $\mathfrak{p}_{i}$ contains one of the prime ideals belonging to $I(C)$. Since $\operatorname{dim} I(C)=n-3$ its prime ideals all have dimension $\leqq n-3$, and we will have shown that this is impossible if we can show that $\operatorname{dim} \mathfrak{p}_{i}=n-2$. Since $\left\langle c_{1}, c_{2}\right\rangle=1,\left(c_{1}, c_{2}\right)$ is not a principal ideal (it is not $R$ because $I(C)$ is not $R$ ) and so has dimension $\leqq n-2$, i.e., all its prime ideals have dimension $\leqq n-2$. Then by the theorem of van der Waerden 
$[5$, p. 70$]$ it follows that its prime ideals all have dimension actually equal to $n-2$, which is what we needed.

Thus Lemma 5.1 is applicable, and $N=N^{\prime}$. Now suppose $C \in \Re$. Then $\exists \bar{a}, b \in N$, and hence in $N^{\prime}$, whose coordinates satisfy equations (1), (2), and (3) of $\S 3$. Since $\bar{a} \in N^{\prime}, a_{1} \in\left(c_{2}, c_{3}\right) \subseteq I(C)$, and similarly all the $a_{i}$ 's and $b_{i}$ 's are in $I(C)$. Then equations (1), (2), and (3) imply that all the $c_{i}$ 's are in $I(C)^{2}$; hence $I(C) \subseteq I(C)^{2}$, and so $I(C)=I(C)^{2}$. Then it follows by induction that $I(C)=I(C)^{n}$ for all $n$, and hence that $I(C)=\bigcap_{n=1}^{\infty} I(C)^{n}$. But this last is impossible, since $I(C) \neq R$ implies that $\bigcap_{n=1}^{\infty} I(C)^{n}=(0)$.

Two matrices will be said to be equivalent (in the sense of this paper) if one is a commutator if and only if the other is, and a $2 \times 2$ matrix $C$ of trace 0 will be said to have dimension $k$ if $I(C)$ has dimension $k$. We recall that an ideal is called unmixed or mixed according as its prime ideals do or do not all have the same dimension.

We can show that every matrix of dimension -1 is equivalent to a matrix of unmixed dimension $n-2$. For let $C$ have dimension -1 , so that $\left(c_{1}, c_{2}, c_{3}\right)$ $=(1)$. We claim that there exists a prime polynomial $d$ which is not a factor of either $c_{1}$ or $c_{3}$, and which has at least one zero in common with $c_{1}$. To verify this it will certainly be enough to show that there are infinitely many prime polynomials which have zeros in common with $c_{1}$, and this is an immediate consequence of the fact that the manifold of $c_{1}$ contains infinitely many points. Thus there exists a $d \in R$ such that $\left\langle d, c_{1}\right\rangle=\left\langle d, c_{3}\right\rangle=1$ and $\left(c_{1}, d\right)$ $\neq(1)$. Then $\left(c_{1}, d\right)$ cannot be a principal ideal, i.e., cannot have dimension $n-1$, and so must have dimension $n-2$. By the theorem on p. 70 of van der Waerden [5] it follows that $\left(c_{1}, d\right)$ is actually an unmixed $n-2$ dimensional ideal. Furthermore, since $1 \in\left(c_{1}, c_{2}, c_{3}\right), d \in\left(d c_{1}, d c_{2}, d c_{3}\right) \subseteq\left(c_{1}, d c_{2}, d c_{3}\right)$, so that $\left(c_{1}, d c_{2}, d c_{3}\right)=\left(c_{1}, d\right)$. Now since $\left\langle c_{1}, d\right\rangle=1, C$ is equivalent, by Lemmas 3.3 and 3.9, to the matrix

$$
\left(\begin{array}{cc}
c_{1} & d c_{2} \\
d c_{3} & -c_{1}
\end{array}\right)
$$

which in turn is equivalent, by Lemmas 3.8 and 3.1 , to

$$
M=\left(\begin{array}{cc}
c_{1} & c_{1} c_{3}+d c_{2} \\
d c_{3} & -c_{1}
\end{array}\right) .
$$

But $I(M)=\left(c_{1}, d c_{2}, d c_{3}\right)=\left(c_{1}, d\right)$ is an unmixed $n-2$ dimensional ideal, and so to complete the proof we need only show that no two entries in $M$ have a common factor. The verification of this is straightforward.

Thus the problem reduces to an investigation of the matrices of dimension $n-2$. This is more complicated; it is easy to produce examples of such matrices which are commutators and of others which are not. However, all examples known to date behave as follows: If $I(C)$ is a mixed $n-2$ dimen- 
sional ideal then $C$ is not a commutator, while if $I(C)$ is unmixed then $C$ is a commutator. For example, it is easy to show that the matrices in which one entry is a linear polynomial can be classified in this way. If this were known to be true in general the problem would be solved; in particular we would know Theorem $\mathrm{C}$ (and hence Theorem A) to be true, since Theorem $\mathrm{C}$ is just the special case for which the dimension is -1 .

It is tempting to conjecture at this point that the property of being a commutator is a geometric property of the matrix $C$ in the sense that it does not depend on the ideal $I(C)$, but only on its manifold. We will give a specific example to show that this is not the case. We take $R$ to be $K[x, y, z]$, let $V(C)$ and $V(a, b)$ denote the manifolds of the matrix $C$ and the ideal $(a, b)$ respectively, and let

$$
M=\left(\begin{array}{cc}
x & y z \\
x+y^{2} & -x
\end{array}\right) \text { and } M^{\prime}=\left(\begin{array}{cc}
x & y \\
x+y & -x
\end{array}\right) .
$$

Then $V(M)=V\left(x, y z, y^{2}\right)=V(x, y)=V\left(M^{\prime}\right)$. Certainly $M^{\prime} \in \mathcal{K}$, since $x+y$ $\epsilon(x, y)$. Now by Lemmas $3.3,3.8$, and $3.9, M$ is equivalent to

$$
\left(\begin{array}{cc}
x & y z \\
y^{2} & -x
\end{array}\right)
$$

which in turn is equivalent to

$$
\left(\begin{array}{rr}
x & z \\
y & -x
\end{array}\right)
$$

and this is certainly not a commutator, $\operatorname{since} \operatorname{dim}(x, y, z)=0=n-3$. The trouble, of course, is that although $I(M)$ has an unmixed manifold, it is a mixed ideal; in fact

$$
I(M)=\left(x, y z, y^{2}\right)=\left(x, z, y^{2}\right) \cap(x, y) .
$$

The fact that the matrix

$$
\left(\begin{array}{rr}
x & y \\
z & -x
\end{array}\right)
$$

is not a commutator can be shown directly in a quite elementary way; we will do this, since the method leads to some interesting generalizations. Suppose

$$
\left(\begin{array}{rr}
x & y \\
z & -x
\end{array}\right)
$$

is a commutator; then $\exists a_{i}, b_{i} \in R$ satisfying 


$$
\begin{aligned}
& x=a_{2} b_{3}-b_{2} a_{3}, \\
& 0=a_{1} x+a_{2} z+a_{3} y,
\end{aligned}
$$

and

$$
0=b_{1} x+b_{2} z+b_{3} y .
$$

Now we claim it follows from the last equation that the polynomial $b_{1}$ has no constant term, for if it had, the product $b_{1} x$ would have a linear term in $x$, whereas the expression $b_{2} z+b_{3} y$ clearly has not. Similarly $b_{2}$ and $b_{3}$ do not have constant terms either, and neither do any of the $a_{i}$ 's. But then the products $a_{2} b_{3}$ and $b_{2} a_{3}$ can have no linear terms, so that the first equation above is not possible.

Now let $R=K\left[x_{1}, \cdots, x_{n}\right]$ again, where $n \geqq 3$. Then the natural generalization of the above argument gives

LEMMA 5.2. Let

$$
C=\left(\begin{array}{rr}
c_{1} & c_{2} \\
c_{3} & -c_{1}
\end{array}\right),
$$

where each $c_{i}=x_{i}+p_{i}$, the $p_{i}$ 's being polynomials with no constant terms and no linear terms in $x_{1}, x_{2}$, and $x_{3}$. Then $C \notin \mathcal{K}$.

As a consequence of this, we can now prove

Theorem 5.2. For any field $K$, Theorem B is false for $K\left[x_{1}, \cdots, x_{n}\right]$ if $n \geqq 6$.

Proof. We claim there does not exist a matrix with first row $\left(x_{4}, x_{5}, x_{6}\right)$ and determinant $=d=x_{1} x_{4}+x_{2} x_{6}+x_{3} x_{6}$, although certainly $d \in\left(x_{4}, x_{5}, x_{6}\right)$. Suppose such a matrix does exist, i.e., $\exists a_{i}, b_{i}$ such that

$$
\left|\begin{array}{lll}
x_{4} & x_{6} & x_{5} \\
a_{1} & a_{2} & a_{3} \\
b_{1} & b_{2} & b_{3}
\end{array}\right|=x_{1} x_{4}+x_{2} x_{5}+x_{3} x_{6}
$$

Then we have $x_{4} \alpha+x_{5} \beta+x_{6} \gamma=0$, where $\alpha=a_{2} b_{3}-b_{2} a_{3}-x_{1}, \beta=a_{1} b_{2}-b_{1} a_{2}-x_{2}$, and $\gamma=a_{3} b_{1}-b_{3} a_{1}-x_{3}$. Now certainly $\left(x_{4}, x_{5}\right)$ is a prime ideal, and $x_{6} \notin\left(x_{4}, x_{5}\right)$, so the hypothesis of Lemma 5.1 is satisfied, and we can conclude that the vector $(\alpha, \beta, \gamma) \in N^{\prime}$. Then $\exists K_{i} \in R$ such that $\alpha=K_{1} x_{5}+K_{2} x_{6}, \beta=-K_{1} x_{4}$ $+K_{3} x_{6}$, and $\gamma=-K_{2} x_{4}-K_{3} x_{5}$. This gives us

$$
\begin{aligned}
& a_{2} b_{3}-b_{2} a_{3}=x_{1}+K_{1} x_{5}+K_{2} x_{6}, \\
& a_{1} b_{2}-b_{1} a_{2}=x_{2}-K_{1} x_{4}+K_{3} x_{6},
\end{aligned}
$$

and

$$
a_{3} b_{1}-b_{3} a_{1}=x_{3}-K_{2} x_{4}-K_{3} x_{5} .
$$


But the three polynomials appearing on the right-hand side of these equations satisfy the hypothesis of the previous lemma, hence do not form a commutator, and so these equations cannot be satisfied.

For formally real fields we can sharpen this to

THEOREM 5.3. For any formally real field $K$, Theorem B is false for $K\left[x_{1}, \cdots, x_{n}\right]$ if $n \geqq 3$.

Proof. Now we show that there does not exist a matrix with first row $\left(x_{1}, x_{2}, x_{3}\right)$ and determinant $=x_{1}^{2}+x_{2}^{2}+x_{3}^{2}$. It follows just as before that if such a matrix does exist then $\exists a_{i}, b_{i}$ satisfying

$$
x_{1}\left(a_{2} b_{3}-b_{2} a_{3}-x_{1}\right)+x_{2}\left(a_{1} b_{2}-b_{1} a_{2}-x_{2}\right)+x_{3}\left(a_{3} b_{1}-b_{3} a_{1}-x_{3}\right)=0,
$$

and then we can again conclude that $\exists K_{i} \in R$ such that

$$
\begin{aligned}
& a_{2} b_{3}-b_{2} a_{3}=x_{1}+K_{1} x_{2}+K_{2} x_{3}, \\
& a_{1} b_{2}-b_{1} a_{2}=x_{2}-K_{1} x_{1}+K_{3} x_{3}, \\
& a_{3} b_{1}-b_{3} a_{1}=x_{3}-K_{2} x_{1}-K_{8} x_{2} .
\end{aligned}
$$

Now let $f_{i}$ denote the constant term in $K_{i}$, and let

$$
\begin{aligned}
& x_{1}^{\prime}=x_{1}+f_{1} x_{2}+f_{2} x_{3}, \\
& x_{2}^{\prime}=-f_{1} x_{1}+x_{2}+f_{3} x_{3}, \\
& x_{3}^{\prime}=-f_{2} x_{1}-f_{3} x_{2}+x_{3} .
\end{aligned}
$$

We wish to express the $x_{i}^{\prime}$ 's as functions of the $x_{i}^{\prime \prime}$ s. The determinant of the above set of equations is

$$
\left|\begin{array}{rrr}
1 & f_{1} & f_{2} \\
-f_{1} & 1 & f_{3} \\
-f_{2} & -f_{3} & 1
\end{array}\right|=1+f_{1}^{2}+f_{2}^{2}+f_{3}^{2} \neq 0,
$$

since $K$ is a formally real field. Thus the above set of equations can be solved, i.e., each $x_{i}$ may be expressed as a linear combination of the $x_{i}^{\prime \prime}$ 's with coeffcients in the base field $K$. Now let $x_{i}^{\prime}=x_{i}$ for $i>3$. Then certainly $K\left[x_{1}, \cdots, x_{k}\right]=K\left[x_{1}^{\prime}, \cdots, x_{k}^{\prime}\right]$, i.e., every polynomial in the $x_{i}$ 's can be expressed as a polynomial in the $x_{i}^{\prime}$ 's. Moreover, when we do this a homogeneous polynomial of degree $r$ in the $x_{i}$ 's becomes a homogeneous polynomial of the same degree in the $x_{i}^{\prime \prime}$ 's, so that the degrees of all terms are preserved. Now we note that

$$
\begin{aligned}
& x_{1}+K_{1} x_{2}+K_{2} x_{3}=x_{1}^{\prime}+p_{1}, \\
& x_{2}-K_{1} x_{1}+K_{3} x_{3}=x_{2}^{\prime}+p_{2},
\end{aligned}
$$

and 


$$
x_{3}-K_{2} x_{1}-K_{3} x_{2}=x_{3}^{\prime}+p_{3},
$$

where the $p_{i}$ 's are polynomials in the $x_{i}$ 's with no constant or linear terms. Hence when we express the $p_{i}$ 's as polynomials in the $x_{i}^{\prime}$ 's they still have no constant or linear terms, so that the hypothesis of Lemma 5.2 is again satisfied, and we can again conclude that equations (6) are impossible.

The basic argument we have been using can also be generalized to $n \times n$ matrices. In this case it yields

Theorem 5.4. Let $R=K\left[x_{1}, \cdots, x_{k}\right], k \geqq 2 n-1$. Then there are $n \times n$ matrices over $R$ of trace 0 which are not commutators.

Proof. Let $A=\left(a_{i j}\right), B=\left(b_{i j}\right), C=\left(c_{i j}\right)$, and suppose that $C=[A, B]$. Then

$$
\operatorname{tr}(B C)=\operatorname{tr}(B(A B-B A))=\operatorname{tr}(B A B-B B A)=\operatorname{tr}([B A, B])=0,
$$

so

$$
\begin{aligned}
& \sum_{i=1}^{n} \sum_{j=1}^{n} b_{i j} c_{j i}=0, \quad \text { and similarly } \\
& \sum_{i=1}^{n} \sum_{j=1}^{n} a_{i j} c_{j i}=0
\end{aligned}
$$

Now define $C$ as follows:

$$
\begin{array}{ll}
c_{k 1}=x_{k} & \text { for } 1 \leqq k \leqq n, \\
c_{1 k}=x_{n+k-1} & \text { for } 2 \leqq k \leqq n, \\
c_{k j}=x_{k} & \text { for } 2 \leqq k, j \leqq n \text { and } k+j \neq 2 n,
\end{array}
$$

and

$$
c_{n n}=-\sum_{k=1}^{n-1} x_{k}^{k}
$$

Then $\operatorname{tr}(C)=0$, and we claim that $C \notin \mathcal{K}$.

Suppose $C=[A, B]$; we know we may assume $a_{n n}=b_{n n}=0$, so equations (7) become

$$
0=\sum_{k=1}^{n} b_{1 k} x_{k}+\sum_{k=1, j=2 ; i+j \neq 2 n}^{n} b_{k 1} x_{n+k-1}+b_{i j} x_{j}^{i}
$$

and

$$
0=\sum_{k=1}^{n} a_{1 k} x_{k}+\sum_{k=2}^{n} a_{k 1} x_{n+k-1}+\sum_{i, j=2 ; i+j \neq 2 n}^{n} a_{i j} x_{j}^{i} .
$$

Now we are ready to apply the familiar argument. It follows from the first of these equations that the polynomial $b_{1 k}$ can have no constant term, for if it 
had the product $b_{1 k} x_{k}$ would have a linear term in $x_{k}$, and linear terms in $x_{k}$ cannot occur anywhere else in this equation. Similarly the $b_{k 1}$ 's, $a_{k 1}$ 's and $a_{1 k}$ 's can have no constant terms. Now we have

$$
x_{1}=c_{11}=\sum_{k=1}^{n} a_{1 k} b_{k 1}-\sum_{k=1}^{n} b_{1 k} a_{k 1}
$$

but none of the products occurring on the right can have linear terms, so this is impossible.

\section{BIBLIOGRAPHY}

1. D. G. Northcott, Ideal theory, Cambridge, University Press, 1953.

2. J.-P. Serre, Faisceaux algebriques coherrents, Ann. of Math. vol. 61 (1955) pp. 197-278.

3. C. S. Seshadri, Triviality of vector bundles over the affine space $K^{2}$, Proc. Nat. Acad. Sci. U.S.A. vol. 44 (1958) pp. 456-458.

4. B. L. van der Waerden, Modern algebra, Vol. I, New York, Frederick Ungar Publishing Co., 1949.

5. - Modern algebra, Vol. II, New York, Frederick Ungar Publishing Co., 1950.

6. Séminaire Paul Dubreil et Charles Pisot, Paris Université, vol. 2, 1957-1958.

Cornell University, ITHACA, NEW YORK

NORTHWESTERN UNIVERSITY

Evanston, Illinois 